\title{
A Scoping Review of Telehealth
}

\author{
P. A. Abbott' ${ }^{1}$ Y. Liu ${ }^{2}$ \\ ' University of Michigan School of Nursing, Ann Arbor, MI, USA \\ ${ }^{2}$ University of Michigan School of Information, Ann Arbor, MI, USA
}

\begin{abstract}
Summary
Objectives: This scoping review of the telehealth literature over

the past year was conducted to provide a snapshot of some of the current developments in the field. As with any scoping review, only a subset of papers was examined, and the rigorous methods of a systematic review are not applied.

Methods: We surveyed selected dimensions of the current literature, specifically targeting telehealth or eHealth interventions at the patient (or micro) level in this scoping review. Considering the lack of clarity around the terms like mHealth, eHealth, telehealth, and telemedicine, efforts were made understand and harmonize the terminology as part of the review process. Results: A total of 171 papers that matched the search criteria were culled from the literature. After discussion and debate, a total of 26 papers were retained and classified into at least one of 5 conceptual categories that were derived form a concept analysis. The five categories are Preventive and Therapeutic Effects; Health Service Utilization; Challenges \& Opportunities for Enhanced User Centered Design; Low-powered studies/inconclusive evidence; and Future trends in telehealth. Each of these 5 concept categories are discussed to provide a better understanding of present opportunities, challenges, and the overall prospects for telehealth advancement.

Conclusions: The field is expanding and maturing rapidly. There is a need for larger scale studies that balance rigor while reducing translational latency. Additional attention to implementation science methods is recommended as global telehealth projects accelerate.
\end{abstract}

\section{Keywords}

Telehealth, eHealth, implementation science, mHealth, global health

Yearb Med Inform 2013:51-8

\section{Introduction}

Improving quality, safety, and efficiency of healthcare is a clarion call for a variety of global stakeholders. Policymakers, insurers, researchers, patients and their families, clinicians, and numerous others have joined the effort to make the achievement of good health, and access to quality healthcare, a basic human right. The path to achieving such lofty goals however is convoluted and difficult. In addition to the seemingly intractable issues of poverty, conflict, humanitarian emergencies, infectious diseases, and inequity that interfere with the achievement of higher levels of health, we are also burdened by accelerating costs, an aging global population, and marked escalations in chronic diseases.

In the face of such considerable impediments, some may view the glass as half-empty or simply too overwhelming to address in total. Others view these challenges from a different and more positive perspective, capitalizing on the confluence of health consumerism, patient-centered decision-making, and increasing global digitization. Indeed, the marked growth of global eHealth is evidence of new consideration being given to both the opportunities that are present, and the challenges that need new approaches to be solved.

This scoping review is designed to survey selected dimensions of the current literature, specifically targeting telehealth or eHealth interventions at the patient or micro level. Literature at the larger macro level (i.e. populations and organizations) was also scanned with emphasis given to findings that relate to outcomes at the individual patient level. The review was no easy task; literature about eHealth, telehealth, mHealth and similar was copious in the one year time period of this review, hence our necessary narrowing of focus and our need to define and match the terminology. As it is characteristic of a scoping review [1] the mapping and judging of the evidence in this review is conducted without extensive data synthesis or the rigor of a full systematic review.

\section{Scoping}

\section{Coming to Terms with the Terminology}

What is meant by the term eHealth? Is eHealth the same as mHealth and as telehealth or telemedicine? Can we use the same terms to survey the literature with any sense of assurance that the underlying concepts and meanings are the same? In scanning the literature to answer these questions, the terms mHealth, eHealth, telehealth and telemedicine were found to be used interchangeably - giving rise to a primary concern (echoed by many) that the lack of clarity around the "label" makes the evidence base more difficult to assess. One of our goals in this survey therefore was to understand and harmonize the terminology to the degree possible - allowing us to discern differences when considering results.

In the process of closer examination of the publications, we concluded that while these terms are common in many ways and have some of the same origins, there are distinct differences. Telemedicine, the grandfather term, is defined as "the use of medical information exchanged from one site to another via electronic communications to improve a patient's clinical health status" [2]. Formally originating in the 1970s, telemedicine was viewed as a way to provide health services to remote patients, be they in a space shuttle or in a frontier community. More current adaptations of the term telemedicine have 
attempted to incorporate mHealth or "mobile health" and eHealth (electronic health) as components of the domain of telemedicine.

Telehealth or eHealth were viewed as expansions of the telemedicine term in many of the publications that we reviewed. These two terms began gaining in popularity as the application space moved away from one perceived as medical or clinical to one that included the broader concept of health with a patient or community centric dimension [3]. EHealth was defined in an article titled "What is eHealth: The death of telemedicine?" [4], as "a new term needed to describe the combined use of electronic communication and information technology in the health sector... the use in the health sector of digital data - transmitted, stored and retrieved electronically - for clinical, educational and administrative purposes, both at the local site and at distance." Defined by the European Commission as the "overarching term for the range of tools based on information and communication technologies used to assist and enhance the prevention, diagnosis, treatment, monitoring, and management of health and lifestyle" [5], eHealth as a term has been adopted by the World Health Organization (WHO). Since the adoption of the $58^{\text {th }}$ World Health Assembly's WHA58.28 eHealth Resolution [6], 72 national eHealth strategies have been developed and implemented globally [7]. WHO now incorporates the terms telehealth, telecare, and telemedicine under the term eHealth.

mHealth, as a subset of eHealth or telehealth, is a more current adaptation of the terminology, and is defined as "the use of mobile and wireless devices to improve health outcomes, healthcare services, and health research" [8]. A contributor to what makes mHealth "new" (and one of the major drivers for the recent massive upticks in mHealth interventions) is the rapidly developing global telecommunications infrastructure and the availability of increasingly powerful and affordable cellular telephony in the hands of the general population. Innovative applications in this space are multiplying, rapidly leveling the playing field, increasing the concept of "patient as partner", and extending opportunity for improving health to the farthest reaches of the globe [9].
A recent literature review pertinent to this terminology discussion conducted by Fatehi $\&$ Wooten [10] examined the use of the commonly associated terms of telehealth, eHealth, and telemedicine from the years 1972 to 2011, asserting that the most appropriate term to represent this domain is "eHealth". Based on these assertions and for the purposes of this scoping review, we will use the overarching term of eHealth $(\mathrm{eH})$ for general reference in this review. However, we will use the terms telemedicine and mHealth where required (i.e. if the paper focuses upon that term exclusively). As Piette et al. point out [11], there are many different areas of impact of eHealth, from supply chain management at a national level to the relationship between SMS'ing and an individual patient's ability to stop smoking. As noted in the introduction to this paper we chose to focus upon literature that spoke to the impact at a more granular patient level, rather than examining large scale interventions at a general population level.

\section{Methods}

Mimicking the approach used by Piette et al.[11], a scoping review was conducted using targeted databases with further investigation and retention of articles derived from the retained article reference lists (snowballing). A MEDLINE search was conducted, using the terms "research", "trends", "systematic reviews", and "literature reviews" in combination with "mHealth", "eHealth", "telehealth", and "telemedicine". We focused on articles whose outcomes were at a patient level. Articles that were focused upon eHealth at a larger, "systems" level (i.e. eHealth for national supply chain management, surveillance, hospital management, systems that aggregate data for population level interventions, and the like) were scanned and considered for applicability or additional references. In addition, based on the experience of the authors of this review and advice from other similar experts, additional sources were identified and searched. In example, recorded keynote presentations, book chapters, and national eHealth strategies accessible from the WHO were also scanned for relevancy.
Considerations and Caveats of the Methods Used - To provide a fair approach to an examination of the literature related to $\mathrm{eH}$ at the level of the individual, we acknowledged the requisite balancing act of "not letting perfect being the enemy of the good." In example, the literature is replete with criticisms of inconclusive evidence of eH impact, insufficient power/small sample sizes which threatens the findings (referred to as "pilotitis"), lack of definition clarity, weak study design, and calls for additional research conducted at a higher level of rigor. At the same time, there are pleas (to the scientific community from those who are in dire need of eHealth interventions, particularly in developing nations) to avoid the extended periods of latency associated with traditional research studies. Hamadoun Touré, General Secretary for the International Telecommunications Union at the January 2013 GetHealth Summit at the UN made the point that the often cited 17 years from bench to bedside "will doom an entire generation" [12].

In essence, the take away here, may be one of balancing on the edge of a razor; while many calls for a cure for "pilotitis" in eH interventions, the costs associated with the deployment of questionable telehealth applications is one that many regions simply cannot afford. These arguments raise the specter of the "evaluation paradox" discussed by Friedman and Wyatt [13], where the point is made that the "intervention" has an impact at multiple levels, thereby making it quite difficult to completely assess the full impact of the change across varying levels. In example, telehealth interventions for chronic disease management often affect patients and the organization at the same time - there are dimensions of behavioral modification from the patient's perspective, and organizational impacts from decreased use of acute care services. Similar issues with differentiating levels of impact are raised by Van de Ber et al. [28], and are discussed later in this paper. The point is that the evaluation paradox in eH studies is common and can muddy results and interpretations. These considerations do impact this scoping review.

Therefore, we believe that there is a balance to be struck in regards to a "good enough" study versus a randomized clinical 
trial; between effects on individual patients versus systems or organizational impacts; and the methods used to characterize the patient outcomes in regards to disease prevention, health promotion, user satisfaction, and the like. In addition, considering eHealth in the context of developing nations interjects tremendous variation. While the intent of this review is to provide a transparent and broad map of a variety of current literature, the interpretation of this work is sensitive to context, interpretation, and the methodology of a scoping review.

\section{Results}

The MEDLINE search of "telehealth" "telemedicine" "ehealth" "mhealth" combined with the terms "research", "trends", "systematic reviews", and "literature reviews" from 12/31/2011 to 12/31/2012 resulted in a total of 171 papers. Papers were reviewed based on the criteria discussed earlier in this review. After discussion and debate between the authors of this paper, a total of 26 papers were retained for the scoping review.

In the 26 papers retained, a concept analysis conducted by the two authors of this review resulted in the emergence of 5 major categories. Each of the 26 papers were classified into at least one of the 5 categories of Preventive and Therapeutic Effects (which includes Patient Health Outcomes, Disease Prevention, Symptom Management, \& Disease Management); Health Service Utilization; Challenges \& Opportunities for Enhanced User-Centered Design (including studies related to designing for the user base to improve satisfaction, sensitivities to culture, literacy, affordability and similar); Low-powered studies/inconclusive evidence (Studies that suggested new methodologies and additional studies for investigation of $\mathrm{eH}$ interventions); and Future trends in $\mathrm{eH}$. There are significant overlaps between the categories; therefore papers could fall into more than one category. It is important to note that the categorization is subjective and somewhat arbitrary. In example, separating findings from the User-Centered
Design category and the Preventive and Therapeutic Effects category was difficult and contentious, with a residual feeling of artificiality. In example, there was strong debate on how Health Services Utilization could be truly separated from Disease Management (a sub-category of Preventive and Therapeutic effects). A criticism of poor design and low power was rampant across nearly all of the studies, with two of the studies focused upon this dimension. The analysis of the literature warranted a separate section, although it was a common thread across almost $100 \%$ of the studies. The point is that there is no way to cleanly separate these categories that arose from the concept analysis - we relied on consensus, negotiation, and rationalization in regards to classification. Each of these 5 concept categories derived from the analysis are discussed.

\section{Discussion}

\section{Preventive and Therapeutic Effects Category}

The preventive and therapeutic effects category was associated with the largest number of retained articles (sixteen in total), primarily focused in chronic disease management, health promotion, and symptom management. Seven of the sixteen articles in this category were broad systematic reviews [14-20].

The sub-category of chronic disease management was the most prevalent, with four articles focused primarily on $\mathrm{eH}$ in diabetes management [14, 15, 21, 22], three in mental health [18, 23, 24], two in heart failure [25, $26]$, and two in chronic lung conditions such as asthma and COPD [17, 27]. Two articles $[20,28]$ were focused generically on chronic diseases.

Articles which fell into the sub-category of symptom management \& health promotion included a systematic review on mobile approaches to the impact of SMS'ing on smoking cessation (classified as health promotion) [19], and a literature review regarding $\mathrm{eH}$ methods for pain symptom management [29]. Finally, the remaining article (classified into the category of preventive and therapeutic effects) was a single meta-analysis [16] that focused upon telemedicine and the impact on patient-level mortality in an ICU.

Chronic Disease Management Sub-Category: Chronic diseases in the retained literature included diabetes, mental health, heart failure and COPD/asthma. Each had distinct attributes and findings that can be unique to the application of $\mathrm{eH}$ in these disease populations; short overviews follow.

In regards to the articles that focused upon $\mathrm{eH}$ in diabetic patients $[13,14,20,21]$, the themes of disease self-management and increased patient engagement were common across all. The systematic review of diabetes management via mobile phones by Holtz \& Lauckner [15] also showed positive trends in regards to helping patients to better manage their disease. However, the results varied widely and many of the studies included in their review were reported to be underpowered. Cassimatis \& Kavanagh's systematic review of behavioral telehealth interventions in Type 2 diabetes [14] showed evidence of improved outcomes such as increased medication adherence, adherence to diet plans, and increased physical activity when telehealth approaches were used. Two of the four articles $[21,28]$ related to diabetes specifically contribute perspectives that are additive to the prior positive findings of improved disease self-management, adherence, and the like. First, in the literature review of telemedicine and ocular health in diabetes mellitus by Bursell and colleagues [21], an insightful point is made that "the single act of patients viewing their own retinal images improves self-management behavior and clinical outcomes" (pg. 311). This interesting assertion suggests that patients who are engaged with their own health data, educated in regards to the meaning of that data, and who understand the relationship of the data to their personal health are more prone to alter their behavior in a beneficial way. While the counter-argument could be that $\mathrm{eH}$ is not the only way in which data and images can be shared with patients, the points made by Bursell et al. are in relation to a wider-reaching concept of coordinated, patient-centered, and shared care in a patient-centered digital ecosystem. We found this statement by Bur- 
sell et al. to be supportive of patient-engagement in disease management programs in a way that is a step above simple prescriptive approaches. What are the mechanisms at work behind Bursell's assertions that simply showing a patient their retinal images can actually impact self-management? A deeper understanding here could be an important component of enhanced design of eH applications for chronic disease patients.

The second of the two articles that adds an interesting perspective is the systematic review by van de Berg et al. [28] which focuses on chronic disease in general and CHF specifically. When this work is considered in concert with that of Piette [11], the differentiation of the levels of $\mathrm{eH}$ impact and directions for future studies become more clear. Van de Berg and colleagues assert that there is a trend towards "better results" when eH is used to affect behavioral endpoints (such as medication adherence, weight control, and self-efficacy) versus trying to quantify the impact of $\mathrm{eH}$ on medical outcomes such as mortality, financial outcomes (i.e. cost reduction), and quality of life [28]. This is an area worthy of debate and further investigation. Are the effects of eH on behavioral aspects "better" than those exerted on medical outcomes as van de Berg et al. suggest or is this a classic example of Friedman and Wyatt's [12] evaluation paradox?

The other studies regarding chronic disease management included telehealth for asthma or COPD $(17,27)$, cardiac conditions [25, 26, 30], and the use of telehealth approaches in the provision of mental health services [18, 24]. Of particular interest, the systematic review by Huckvale et al. [27] in regards to the assessment of available $\mathrm{eH}$ "apps" for asthma was very illuminating. Of the 72 instances where apps made explicit recommendations or treatment suggestions, the researchers determined that 32 were unsupported by current evidence. Huckvale et al. also found that, in the 103 total apps reviewed, 56 contained information about asthma and 47 provided tools for the management of asthma, but none of the 103 contained both. This study in particular raises concerns about the safety, quality, and comprehensiveness of eH applications currently being used for asthma.
Appropriately, particularly in consideration of the findings of Huckvale et al., a call for additional research and application is raised in a meta-analysis conducted by McClean et al. [17] on the effects of telehealth use by chronic obstructive pulmonary disease (COPD) patients. The meta-analysis found that while rates of hospitalization and emergency rooms visits were significantly less in the telehealth group, there was no discernible effect on mortality. McClean et al. make the point that "substantial aspects of the technology are inconclusive" (pg. 14) and lead the authors to assert that "many electronic tools for remotely helping patients with COPD are now being implemented in the absence of an explicit evidence. Exploration of the existing evidence of the values or risks of these interventions is therefore urgently required" (pg. 4). The "absence of an explicit evidence base" is an example of the common issue of a lack of clinical validity and concerns of inappropriate or misguided use.

The literature centered on telehealth for cardiac patients $[25,26,30]$ did not include any systematic reviews. Two were general literature reviews $[25,26]$ and the third [30] was a single quasi-experimental study. The benefits of the use of telehealth with heart failure patients (specifically referred to as "monitoring") in the meta-analysis conducted by Acosta-Lobos et al. [25] was determined to be considerable when compared with usual care. The authors strongly suggest however that these types of technological interventions should be reserved for use in unstable and higher risk populations and not used indiscriminately.

The potentials for home monitoring of heart failure patients via sensing technologies and other telehealth approaches are reviewed favorably by Bui \& Fornarow [26]. Chiang et al. [30] take another direction in discussing telehealth and heart failure, choosing to focus on the positive impacts on families and caregivers. Telehealth used in concert with CHF patients was found to reduce caregiver burden, enable better management of stress levels, and improve family function.

When one considers the points made in the COPD and asthma literature with the assertions made by Acosta-Lobos et al. [25] the specter of "technology for technology's sake" comes to the fore. While much of the evidence of the benefits of eH is positive, the potential risks, costs, and questions of the reliability of the findings warrant carefully considerations before automatic deployment. Because of the variation and uncertainty surrounding the use of eH in CHF, COPD, and other chronic diseases, the results of these studies should better be viewed as transferrable rather than generalizable.

The final cluster of papers and studies included here are in regards to tele-mental health $[18,23,31]$. The use of eH in the provision of mental health services is well covered in the systematic review conducted by Sucala et al. [18]. Based on the studies reviewed by this group, questions are raised as to the quality of the therapeutic relationship that can be established in a virtual encounter. Ultimately, their systematic review determined that e-therapy is at least equivalent to face to face therapy, but that more research is needed to fully understand the nuances of technology-mediated relationships between therapist and patient. The two remaining articles $[23,31]$ discuss tele-mental health in two challenging populations; substance abusers and vulnerable Native American populations. The take away from these last two articles is that "one size does not fit all" and that each group has very challenging dimensions that require active management - such as countering mistrust, recidivism, aligning with culturally specific healing models and access.

Overall, and in keeping with the acceleration of chronic diseases globally and the increasing shift to self-management of health conditions, there has been a marked uptick in publications and studies in this category. As the use of digital tools continues to move into the global mainstream, it is expected that the use of $\mathrm{eH}$ in chronic disease management will continue to expand. As pointed out by this small sample of papers, there is much work to be done and generalizability is not assured. However, there is sufficient clear promises in the retained literature.

Symptom Management \& Health Promotion Sub-Categoriy: Many fewer articles fell into this sub-category of Preventive and Therapeutic Effects; one that examined the 
impact of SMS'ing on smoking cessation (classified as health promotion) [19], and a literature review regarding $\mathrm{eH}$ methods for pain symptom management [29].

The review of telehealth interventions targeting pain management showed varying levels of positive impacts for patients, but the rigor of many of the studies was questioned [29]. Specific suggestions for comparative effectiveness research and assessment of short and long term benefits afforded by telehealth technologies for pain management were included in the recommendations section of this review. In addition, it was pointed out that there was scant data related to the potential risks of telehealth interventions in broad populations, with a call for additional studies.

The second paper classified into this sub-category was a systematic review by Whitaker et al. [18] focused on smoking cessation via mobile-phone messaging. The studies included in this review primarily used text-based messaging and were directed at the active stage of quitting (not maintaining). The suggestion made by the authors is that additional work should be conducted to study different eH modalities (other than text messaging), the cost-effectiveness of such interventions, the long-term impact on quit rates, and the potential for behavior modification in low income countries. These points are interesting for the global audience - to date much of the work in the mHealth area in low income countries have not focused on the use for non-communicable diseases (NCD). As the toll of NCDs rises around the world, there is opportunity to develop and study eH interventions in the battle against non-communicable diseases, particularly in low and middle income countries whose fragile economies cannot bear the upward spiral of associated costs.

\section{Health Services Utilization}

As discussed earlier, articles that classified into the concept of health services utilization (HSU) were specifically related to patient centric dimensions of HSU. Relating eH to measures of HSU may be more difficult as the variability of patient types, the context, and potential confounders are high. These challenges were raised by all authors of the papers retained in this category, and it echoes the point brought up earlier by Van de Berg et al. [28] that eH interventions may be better suited for individual behavior change efforts than to quantify the impact on HSU.

The studies retained showed that uses of telehealth by chronic disease patients resulted in lower uses of the Emergency Department $[17,24,25]$ while use in an ICU resulted in lower patient mortality rates due to greater and faster access to specialists [20]. One study looked at the impact of SMS reminders for clinic appointments, showing that SMS reminders improved the attendance at clinic as compared to no reminders, and that they were equally effective as phone reminders, with a lower cost [32]. The study by Lewis et al. [16] showed a positive impact on the treatment of acute injuries in areas outside of the reach of traditional trauma centers via telehealth.

Overall, the literature reviewed seemed to point a positive trend in relation to the impact of eH on health services utilization from a patient-level perspective, although authors have reserves to clearly relate $\mathrm{eH}$ interventions with outcomes. Investigations of the nuances that influence the use of health services by patients are warranted, and implementation science approaches may be most appropriate. It is expected that $\mathrm{eH}$ has tremendous potential to reduce utilization of services, engender greater patient engagement, and shift health and healthcare into homes and communities. However, the influence of context and complexity may preclude traditional approaches to the measurement of this dimension.

\section{Challenges \& Opportunities in User-Centered Design}

\section{Challenges}

eHealth can be used to help deliver socially, culturally, and economically competent care. However, more attention is needed to understand challenges in this context. Factors such as language, literacy, incomes, and cultural norms are critical considerations for the design and implementation of $\mathrm{eH}$ interventions. Additional influences arise from the cognitive style of the user, level of comfort with use of eHealth technologies, and individual perceptions and preferences. None of the articles that were retained specifically discussed human factors and usability, which was notable. User-centered design, as a category in the papers that were retained centered on cultural sensitivity, literacy levels, and equitable access.

An example of this emphasis arose in the article "What counts as cultural competency in telehealth interventions? A call for new directions" [33]. Concerns about the lack of debate regarding the dynamic role that an individual's language practices, social relationships and cultural knowledge play in relation to the efficacy of telehealth interventions were raised. The interrelated role of language and society in a patients' adoption of telehealth or eHealth requires deliberation, a point supported by this particular study.

A similar point is made by Collins et al. [34], from the perspective of the type of tools used to determine health literacy for patients to be able to actually utilize the resources provided by eHealth interventions in the first place. These authors reiterate the known points that inadequate or low health literacy inflate healthcare inefficiencies, increase the rates of health services use, and increase mortality risks. The distinct points in relation to eHealth however is that health literacy must be measured appropriately and realistically in order to properly target patient-centered eH interventions. Collins et al. investigated the tools used to measure health literacy and found that there were varying levels of effectiveness based on different contexts of use. The authors also highlighted the importance of assessing computing literacy as a vital dimension of eHealth appropriateness. Collins et al. also suggested that health literacy may be measured adequately with 1 to 3 simple questions from several different HLSQMs (health literacy screening question methodologies).

The take away from Collins et al. [34] is that maximal benefit can be derived from eHealth interventions when they are matched to the user - and while rigorous assessments of health literacy exist in the evidence base - many are not useable in real-world health 
situations. This resurfaces the potential application of implementation science techniques, where flexibility and adaptation to real-world situations are required. The S-TOFHLA (Shortened Test of Functional Health Literacy in Adults) \& the REALM (Rapid Estimation of Health Literacy in Medicine), the two gold standards for the assessment of health literacy in patients are estimated to take 8 and 4 minutes respectively, limiting their use in clinical settings. Using 1-3 questions as suggested by Collins et al. [34] to determine health literacy may be an effective compromise and more in line with real-world situations.

Strategies for recruiting and retaining vulnerable populations in eHealth interventions were raised by Nagler et al. [35]. Emphasis on disparities in access, variations in use and engagement, and dissimilarities in eHealth literacy are specific challenges when working with populations of this type. Additionally, simply providing connectivity and digital equipment is not sufficient. In-person contacts facilitated by accepted members of community groups were shown to help increase health literacy and lessen the digital divide. Moreover, technical and educational support was better accepted when provided by members of the community that are familiar with the challenges and cultural norms of the community. Nagler et al. warn that without the requisite support needed to bridge the digital divide in vulnerable populations, the promise of eHealth may not be realized and the potential for widening the gap is a real possibility.

\section{Opportunities}

Four systematic review papers [23, 36-38] suggest that telehealth is "promising", or has a potential benefit, although the evidence included in their reviews is not powerful enough for the effects to be labeled as causal. Several studies in this cluster of four put forth the belief that telehealth or eHealth has the potential to contribute to user satisfaction and increase patient engagement. Two reports believe that the true promise of $\mathrm{eH}$ technology will come from reaching rural and disconnected populations. The points made in all four of these reviews, while important, are tempered by the criticisms that the evidence base is not strong enough. All four echo the call for additional high rigor research.

An interesting report focused on the opportunities afforded by patient-generated health data [36], was produced for the US Office of the National Coordinator for Health IT. This report produces a comprehensive environmental scan of the impact of data originating from telehealth devices in the hands of patients or from sensing technology. The authors describe the rapid acceleration of patient-generated data and the potential to improve outcomes and enhance patient-provider communication. While this report has many facets, the overall impression is that patient-generated health data will only increase in size, have the potential to increase patient engagement, will force providers into being less patronistic, and ultimately result in improved patient outcomes. A similar theme is raised by Ho et al. [37] who explored the link between the promotion of eHealth, its impact on the public engagement, and the responsibilities of practitioners to move patients from passive recipients to more active and engaged partners in care. Overall, while points are made about the benefit of telehealth, the message of ownership and responsibility (of both patients and providers) emerges as a primary, and critically important, message.

The final two articles reviewed in this category were very specific to certain groups of individuals. The first, entitled "Supporting Family Members of People with Traumatic Brain Injury using Telehealth: a Systematic Review" [38], describes the effectiveness of using telehealth programs to provide training or support to family members of people with traumatic brain injury. This comprehensive work included a review of 7 randomized controlled trials, 4 non-randomized controlled trials, and 5 case series studies. All but one study reported positive outcomes of telehealth programs in regards to satisfaction of family members who interacted with telehealth interventions to support and/or educate families and caregivers. The second article by Shore et al. [23] described the opportunity realized by the provision of high quality and culturally specific tele-mental health care provided to American Indian veterans. A combination of geographic distance, a fundamental mistrust of the US federal system, stigma around mental health, and characteristics of posttraumatic stress disorder (PTSD), added a high level of challenge to the use of eH in these populations. However, this study showed that via targeted and appropriately leveled telehealth services, direct engagement with individuals and communities that trust, rapport, and positive outcomes arose and have been replicated repeatedly in several different regions.

The papers that were reviewed and classified under User-Centered Design illustrated the need for more attention to eH design and implementation based on attention to the end-user. This is particularly a propos when working with vulnerable populations where mistrust and misalignment with cultural norms can make the difference between success and failure of an $\mathrm{eH}$ intervention.

\section{Low-powered Studies / Inconclusive Evidence}

A primary criticism of many of the papers reviewed involved inconclusive evidence, low-powered studies, and a lack of clarity surrounding the definitions of concepts in many of the studies. While we could have classified almost all of the studies retained in this review into this category due to similar criticisms, we chose to specifically classify two of them that were particularly focused on issues of low power and/or low reliability. There is a merit in the points made repeatedly across almost the entire literature according to to low power. Many of the studies show too many that have never grown past a pilot stage where the samples are small and the timeframes exceedingly short. As discussed earlier in this paper, however, this point is contested and somewhat controversial - depending on one's perspectives about pilotitis versus longer and more rigorous studies.

Regardless, the criticisms across many of the systematic reviews included in this paper raise many of the same points, and interject many of the same doubts. In example, Ciere et al. conducted a systematic review of controlled trials and pre-post studies regarding the impact of telehealth on self-care, self-efficacy or knowledge [39]. In this detailed investigation, the authors were 
unable to replicate prior findings, ranked the quality of the studies to be either moderate or poor, and criticized the lack of internal consistency of the studies. Similarly, in the literature review conducted by Young [31] regarding $\mathrm{eH}$ on substance abuse disorder treatment, the criticisms of terms inconsistency, small sample sizes, lack of power, and the introduction of many interventions at one time via $\mathrm{eH}$ (with a single baseline measurement) resulted in studies of low confidence. While the assessment of the potential utility of $\mathrm{eH}$ in the area of mental health service provision was good, these authors' position was that it is difficult to confidently assert the effectiveness of eH taking into account the quality of the results published.

It is obvious that the growth of $\mathrm{eH}$ will continue, particularly as cellular telephony spreads and health needs of the global and aging population spiral upwards. The articles reviewed illustrate the difficult balance between pilotitis, questionable results, and the pressure of a rapidly moving digital health marketplace. It could be said that these are simply the growing pains of a nascent field, however the points are well taken. Additional studies must be undertaken, but at the same time, there must be a pragmatic balance.

\section{Future Trends}

Nearly $100 \%$ of the articles that were included in this scoping review had some elements of "future trends" included. In example, many of the works included ideas for future research, implications for technological advancements such as telecommunications and sensor technology to evolve, and the potential impacts of changing political agendas. The point made by Acosta-Lobos [25] sums up many of the ideas presented in other papers that we reviewed: "Face-toface interaction between patients and their healthcare advisors will not be undermined, but will be employed more at the patient's convenience, and when it is essential for better decision-making and better outcome. Routine 'scheduled' hospital-based appointments are likely to be less frequent, replaced by more remote input into the patient's (self) care unless face-to-face interaction is necessary" (pg. 435). The general article by Paone and Shevchik [40] was retained specifically for its focus on future trends, particularly as related to business trends in $\mathrm{eH}$. These authors posit that business models and reimbursement schemes that address $\mathrm{eH}$ modalities are emerging. In many industrialized nations, marketing information services and information technology for chronic disease populations have become big business. Governments are beginning to shift their attention to health promotion, disease prevention and chronic disease management via $\mathrm{eH}$, particularly in populations for whom they are financially liable [40]. Again, the point is made that there must be much more attention paid to understand the needs of the user and the variations of implementation in real-world environments, especially as the global population ages and the needs for health services increase.

\section{Limitations of the Review}

As noted earlier, this scoping review is not a systematic review and we limited our literature extraction to articles published in 2012. Therefore the characteristic rigor used in a systematic review was not applied here. While this review attempted to produce an overview of current literature related to eHealth or telehealth at an individual patient level, the probability that articles were missed in the searching patterns is moderately high. We did not look at papers that were not in the English language and we were strict about the dates of inclusion and exclusion. Several illuminating articles were unable to be included for these reasons. Finally, we did not have distinct rules about category assignments, and the manner of derivation of the five concepts was based on a consensus between two independent judges, which may threaten the reliability of the concepts.

\section{Conclusion}

In summary, while there are many challenges and growing pains for eH, the overall prospects for growth are very high. Many opportunities exist for focused development and the literature review shows a marked need for additional research. As eH continues to expand, morph, and meld - creative yet rigorous approaches to developing, implementing, and studying must go handin-hand. Implementation science techniques may help to build the knowledge base that is so desperately needed in $\mathrm{eH}$.

\section{References}

1. Armstrong R, Hall BJ, Doyle J, Waters E. Cochrane Update. 'Scoping the scope' of a cochrane review. J Public Health (Oxf) 2011;33(1):147-50.

2. What is Telemedicine? American Telemedicine Assoiciation; n.d. [updated n.d.; cited 2013 Jan 23]; Available from: http://www.americantelemed. org/learn/what-is-telemedicine.

3. Welcome to Telescope: Telehealth Services Code Of Practice for Europe. European Telehealth Code; n.d. [updated n.d.; cited 2013 Feb 1]; Available from: http://www.telehealthcode.eu.

4. Della Mea V. What is e-health (2): the death of telemedicine? J Med Internet Res 2001;3(2):E22. PMCID: 1761900.

5. European Commission eHealth Defined. Accessed 6/1/1013 at: http://ec.europa.eu/health-eu/ care_for_me/e-health/

6. Hogberg U. The World Health Report 2005: "make every mother and child count" - including Africans. Scand J Public Health 2005;33(6):409-11.

7. Progress on eHealth in a Global Context. Pan American Health Organization; n.d. [updated n.d.; cited 2013 Feb 1]; Available from: http://new. paho.org/ict4health/index.php?option=com_content $\&$ view $=$ article $\&$ id $=159 \% 3$ Aavances-de-esalud-en-un-contexto-global\&catid $=42 \% 3 \mathrm{Asec}$ cion-qen-otras-regiones-de-la-omsq\&Itemid $=64$ \&lang=en.

8. What is mHealth? Health Resources and Services Administration; n.d. [updated n.d.; cited 2013 Jan 22]; Available from: http://www.hrsa.gov/healthit/ mhealth.html.

9. Abbott PA, Coenen A. Globalization and advances in information and communication technologies: the impact on nursing and health. Nurs Outlook 2008;56(5):238-46 e2.

10. Fatehi F, Wootton R. Telemedicine, telehealth or e-health? A bibliometric analysis of the trends in the use of these terms. J Telemed Telecare 2012;18(8):460-4.

11. Piette JD, Lun KC, Moura LA, Jr., Fraser HS, Mechael PN, Powell J, et al. Impacts of e-health on the outcomes of care in low- and middle-income countries: where do we go from here? Bulletin of the World Health Organization 2012;90(5):365-72. PMCID: 3341688.

12. Touré HI. GETHealth Global Education \& Technology Health Summit Opening Keynote. International Telecommunication Union; 2013 [updated 2013; cited 2013 Feb 6]; Available from: http://www.itu.int/en/osg/speeches/Pages/2013-02-19.aspx.

13. Friedman CP, Wyatt J, SpringerLink. Evaluation 
methods in biomedical informatics. New York, London: Springer; 2006.

14. Cassimatis M, Kavanagh DJ. Effects of type 2 diabetes behavioural telehealth interventions on glycaemic control and adherence: a systematic review. J Telemed Telecare 2012;18(8):447-50.

15. Holtz B, Lauckner C. Diabetes management via mobile phones: a systematic review. Telemed J E Health 2012;18(3):175-84.

16. Lewis ER, Thomas CA, Wilson ML, Mbarika VW. Telemedicine in acute-phase injury management: a review of practice and advancements. Telemed J E Health 2012;18(6):434-45. PMCID: 3399110.

17. McLean S, Nurmatov U, Liu JL, Pagliari C, Car J, Sheikh A. Telehealthcare for chronic obstructive pulmonary disease: Cochrane Review and meta-analysis. Br J Gen Pract 2012;62(604): e739-49. PMCID: 3481514

18. Sucala M, Schnur JB, Constantino MJ, Miller SJ, Brackman EH, Montgomery GH. The therapeutic relationship in e-therapy for mental health: a systematic review. J Med Internet Res 2012;14(4):e110. PMCID: 3411180.

19. Whittaker R, McRobbie H, Bullen C, Borland R, Rodgers A, Gu Y. Mobile phone-based interventions for smoking cessation. Cochrane Database Syst Rev 2012;11:CD006611.

20. Wilcox ME, Adhikari NK. The effect of telemedicine in critically ill patients: systematic review and meta-analysis. Crit Care 2012;16(4):R127.

21. Bursell SE, Brazionis L, Jenkins A. Telemedicine and ocular health in diabetes mellitus. Clin Exp Optom 2012;95(3):311-27.

22. Harris MA, Hood KK, Mulvaney SA. Pumpers, skypers, surfers and texters: technology to improve the management of diabetes in teenagers. Diabetes Obes Metab 2012;14(11):967-72.

23. Shore J, Kaufmann LJ, Brooks E, Bair B, Dailey $\mathrm{N}$, Richardson WJ, Jr., et al. Review of American Indian veteran telemental health. Telemed J E
Health 2012;18(2):87-94.

24. Steventon A, Bardsley M, Billings J, Dixon J, Doll $\mathrm{H}$, Hirani S, et al. Effect of telehealth on use of secondary care and mortality: findings from the Whole System Demonstrator cluster randomised trial. BMJ 2012;344(jun21 3):e3874-e.

25. Acosta-Lobos A, Riley JP, Cowie MR. Current and future technologies for remote monitoring in cardiology and evidence from trial data. Future Cardiol 2012;8(3):425-37.

26. Bui AL, Fonarow GC. Home monitoring for heart failure management. J Am Coll Cardiol 2012;59(2):97-104. PMCID: 3254025.

27. Huckvale K, Car M, Morrison C, Car J. Apps for asthma self-management: a systematic assessment of content and tools. BMC Med 2012;10:144. PMCID: 3523082.

28. van den Berg N, Schumann M, Kraft K, Hoffmann W. Telemedicine and telecare for older patients--a systematic review. Maturitas 2012;73(2):94-114.

29. McGeary DD, McGeary CA, Gatchel RJ. A comprehensive review of telehealth for pain management: where we are and the way ahead. Pain Pract 2012;12(7):570-7.

30. Chiang LC, Chen WC, Dai YT, Ho YL. The effectiveness of telehealth care on caregiver burden, mastery of stress, and family function among family caregivers of heart failure patients: a quasi-experimental study. Int J Nurs Stud 2012;49(10):1230-42.

31. Young LB. Telemedicine interventions for substance-use disorder: a literature review. J Telemed Telecare 2012;18(1):47-53.

32. Car J, Gurol-Urganci I, de Jongh T, Vodopivec-Jamsek V, Atun R. Mobile phone messaging reminders for attendance at healthcare appointments. Cochrane Database Syst Rev 2012;7:CD007458.

33. Nimmon L, Poureslami I, Fitzgerald JM. What counts as cultural competency in telehealth interventions? A call for new directions. J Telemed
Telecare 2012;18(7):425-6.

34. Collins SA, Currie LM, Bakken S, Vawdrey DK, Stone PW. Health literacy screening instruments for eHealth applications: a systematic review. J Biomed Inform 2012;45(3):598-607. PMCID: 3371171

35. Nagler RH, Ramanadhan S, Minsky S, Viswanath K. Recruitment and Retention for Community-Based eHealth Interventions with Populations of Low Socioeconomic Position: Strategies and Challenges. Journal of Communication 2013;63(1):201-20.

36. Shapiro M, Johnston D, Wald J, Mon D. Patient-Generated Health Data;2012.

37. Ho K, Binfet JT, Cordeiro J, Lauscher HN. Public Engagement in eHealth: Toward Improving Equity in Health Evidence and Health System Renewal; 2012. p. 177-88.

38. Rietdijk R, Togher L, Power E. Supporting family members of people with traumatic brain injury using telehealth: a systematic review. J Rehabil Med 2012;44(11):913-21

39. Ciere Y, Cartwright M, Newman SP. A systematic review of the mediating role of knowledge, self-efficacy and self-care behaviour in telehealth patients with heart failure. J Telemed Telecare 2012;18(7):384-91

40. Paone, S., Shevchik, G. Making a Business Case for eHealth and Teleservices. In: Kumar S, Cohn ER. Telerehabilitation (Health Informatics). Springer; 2012. p. 297-309.

Correspondence to:

Patricia A. Abbott

University of Michigan School of Nursing

Ann Arbor, MI 48109-5482

USA

E-mail: pabbott@umich.edu 